\title{
NOVOS DESENVOLVIMENTOS NUMA FORMULAÇÃO DE INJEÇÃO DE CORRENTE PARA SOLUÇÃO DE FLUXO DE POTÊNCIA
}

\author{
Abilio Manuel Variz* \\ abilio@lacee.ufjf.br
}

\author{
Vander Menengoy da Costa* \\ vandermcosta@uol.com.br
}

${ }^{*}$ UFJF, Faculdade de Engenharia, Juiz de Fora, MG, Brasil

\begin{abstract}
This paper presents a generic methodology of control devices representation into the power flow problem, using a sparse Newton Raphson formulation expressed in terms of current injection equations in rectangular coordinates. This formulation yields the same convergence characteristics presented by power mismatches conventional formulation in polar coordinates. The results validate the proposed methodology.
\end{abstract}

KEYWORDS: Power flow, power systems, current injection, control devices.

\section{RESUMO}

Este artigo apresenta uma metodologia genérica de representação de dispositivos de controle no problema de fluxo de potência, utilizando-se uma formulação Newton Raphson esparsa expressa em termos de equações de injeção de corrente em coordenadas retangulares. Tal formulação possui a mesma trajetória de convergência da formulação convencional em coordenadas polares. Os resultados obtidos validam a metodologia proposta.

PALAVRAS-CHAVE: Fluxo de potência, sistemas de potência, injeção de corrente, dispositivos de controle.

\footnotetext{
Artigo submetido em $13 / 10 / 99$

1a. Revisão em 31/05/00; 2a. Revisão em 10/06/02

Aceito sob recomendação do Ed. Assoc. Prof. Jorge Coelho
}

\section{INTRODUÇÃO}

O cálculo de fluxo de potência é uma ferramenta de suma importância nos estudos de planejamento e operação dos Sistemas Elétricos de Potência. Importantes contribuições nesta área tem sido apresentadas ao longo dos anos (Bacher e Tinney, 1989; Monticelli et alii, 1990). Desenvolvimentos mais recentes incluem a representação de dispositivos de controle no problema de fluxo de potência (Fuerte-Esquivel e Acha, 1997; Gotham e Heydt, 1998; Da Costa et alii, 1998) e outras técnicas de solução (Talaq, 1994; Semlyen, 1996; Da Costa et alii, 1999).

Em Da Costa et alii (1998) são apresentados os modelos matemáticos do transformador defasador, das barras do tipo $\mathrm{P}$ e PQV, do compensador estático de reativo, da compensação série controlada a tiristores, da barra piloto para controle de tensão e finalmente da barra de geração, para a incorporação destes dispositivos de controle no problema de fluxo de potência, utilizando-se uma formulação aumentada ( $4 n x 4 n$ ) expressa em termos das equações de injeção de corrente.

A metodologia convencional de solução de fluxo de potência utiliza as equações de potência expressas em termos das coordenadas polares ou retangulares da tensão nas barras. Em (Da Costa et alii, 1999) é apresentada uma formulação Newton Raphson esparsa para a solução de fluxo de potência, baseada em equações de injeção de corrente expressas em coordenadas retangulares da tensão. A matriz Jacobiana é idêntica à matriz admitância nodal, com exceção dos blocos ( $2 x 2)$ diagonais que são atualizados a cada iteração em função do mo- 
delo de carga adotado. Esta formulação de injeção de corrente vem sendo utilizada com êxito nos cálculos de fluxo de potência para sistemas de distribuição (Garcia et alii, 2000).

O objetivo básico deste trabalho é modelar e implementar de forma eficiente utilizando a formulação de injeção de corrente (2nx2n), os principais dispositivos de controle tratados na solução do problema de fluxo de potência. Nestas modelagens as equações de controle são linearizadas e introduzidas na matriz Jacobiana, obtendose um sistema linear de equações a ser resolvido a cada passo do processo iterativo. Desta forma, tem-se uma maior flexibilidade e robustez matemática na manipulação dos diversos tipos de controle.

\section{NOTAÇÃO}

$n$ : número de barras do sistema

$h$ : contador de iterações

$\Delta \mathrm{P}_{\mathrm{k}}+j \Delta Q_{k}$ :resíduo de potência complexa injetada na barra $k$

$P_{G_{k}}+j Q_{G_{k}}$ :potência complexa gerada na barra $k$

$P_{L_{k}}+j Q_{L_{k}}$ :potência complexa de carga na barra $k$

$P_{k}+j Q_{k}$ :potência complexa injetada na barra $k$

$P_{k}^{s p}+j Q_{k}^{s p}$ :potência complexa especificada na barra $k$ $P_{k}^{\text {calc }}+j Q_{k}^{\text {calc }}$ :potência complexa calculada na barra $k$ $\Delta I_{r_{k}}+j \Delta I_{m_{k}}$ resíduo de corrente complexa na barra $k$ $V_{r_{k}}+j V_{m_{k}}$ :tensão complexa na barra $k$

$V_{k}^{\text {calc }}$ :módulo da tensão calculada na barra $k$

$V_{k}^{s p}$ :módulo da tensão especificada na barra $k$

$\Delta V_{r_{k}}+j \Delta V_{m_{k}}$ :correção da tensão complexa na barra $k$ $G_{k m}+j B_{k m}$ :elemento $(k, m)$ da matriz admitância nodal

\section{FORMULAÇÃO DE INJEÇÃO DE COR- RENTE}

Esta formulação utiliza as equações de injeção de corrente expressas em termos das coordenadas retangulares das tensões, na solução do problema de fluxo de potência. Esta formulação será aqui apresentada de forma sucinta. Maiores detalhes podem ser obtidos em Da Costa et alii (1999).

As equações básicas de corrente numa dada barra $k$ são dadas por:

$$
\begin{aligned}
& \Delta I_{r_{k}}=\frac{P_{k}^{s p} V_{r_{k}}+Q_{k}^{s p} V_{m_{k}}}{V_{r_{k}}^{2}+V_{m_{k}}^{2}}-\sum_{i=1}^{n}\left(G_{k i} V_{r_{i}}-B_{k i} V_{m_{i}}\right)=0 \\
& \Delta I_{m_{k}}=\frac{P_{k}^{s p} V_{m_{k}}-Q_{k}^{s p} V_{r_{k}}}{V_{r_{k}}^{2}+V_{m_{k}}^{2}}-\sum_{i=1}^{n}\left(G_{k i} V_{m_{i}}+B_{k i} V_{r_{i}}\right)=0
\end{aligned}
$$

Aplicando-se o método de Newton Raphson às Equações (1) e (2) considerando-se um sistema genérico onde uma barra $k$ do tipo PV é conectada a uma barra $l$ do tipo PQ, tem-se a seguinte estrutura matricial:

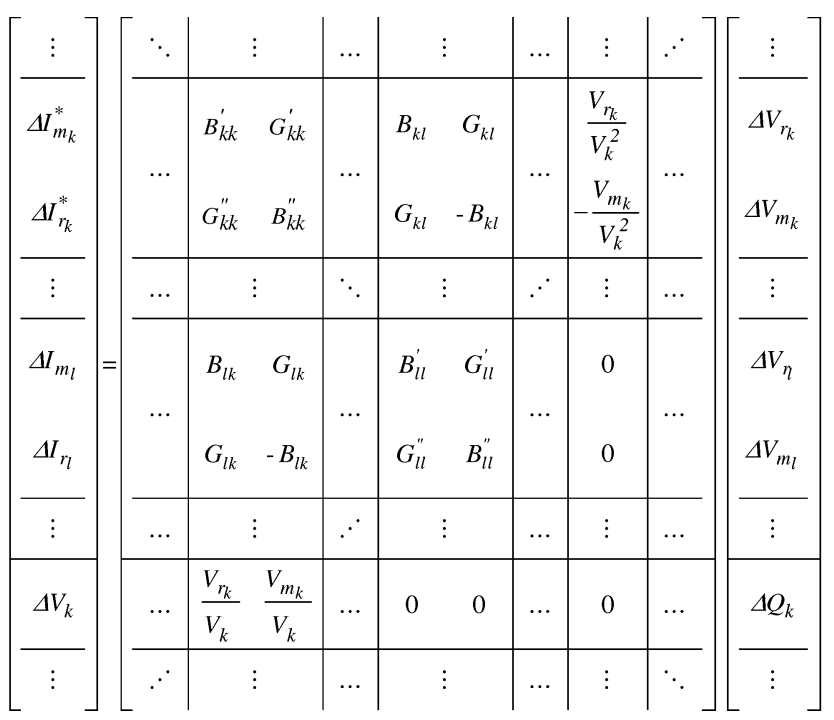

onde:

$$
\Delta V_{k}=V_{k}^{s p}-V_{k}^{c a l c}
$$

Os termos da matriz Jacobiana estão dados em (Da Costa et alii, 1999). Os resíduos de corrente para as barras $l$ e $k$ são dados por: 


$$
\begin{gathered}
\Delta I_{r_{l}}=\frac{V_{r_{l}} \Delta P_{l}+V_{m_{l}} \Delta Q_{l}}{V_{l}^{2}} \\
\Delta I_{m_{l}}=\frac{V_{m_{l}} \Delta P_{l}-V_{r_{l}} \Delta Q_{l}}{V_{l}^{2}} \\
\Delta I_{m_{k}}^{*}=\frac{V_{m_{k}} \Delta P_{k}}{V_{k}^{2}} \\
\Delta I_{r_{k}}^{*}=\frac{V_{r_{k}} \Delta P_{k}}{V_{k}^{2}}
\end{gathered}
$$

Observa-se da Equação (3) que no caso de uma barra $k$ do tipo $\mathrm{PV}$, o resíduo de potência reativa $\Delta Q_{k}$ tornase uma variável dependente. As correções genéricas de tensão em coordenadas polares são dadas por (Da Costa et alii, 1999):

$$
\begin{aligned}
& \Delta V=\frac{V_{r}}{V} \Delta V_{r}+\frac{V_{m}}{V} \Delta V_{m} \\
& \Delta \theta=\frac{V_{r}}{V^{2}} \Delta V_{m}-\frac{V_{m}}{V^{2}} \Delta V_{r}
\end{aligned}
$$

Os valores atualizados do módulo e do ângulo da tensão são dados por:

$$
\begin{aligned}
V^{(h+1)} & =V^{(h)}+\Delta V^{(h)} \\
\theta^{(h+1)} & =\theta^{(h)}+\Delta \theta^{(h)}
\end{aligned}
$$

\section{CONTROLE DE TENSÃO VIA INJE- ÇÃO DE REATIVOS}

\subsection{Controle de Tensão em Barras Remo- tas}

Neste controle a potência reativa de uma barra de geração é usada para controlar o módulo da tensão de uma barra remota. A barra de geração é denominada do tipo $\mathrm{P}$, pois a tensão é desconhecida e somente a potência ativa é especificada. A barra com a tensão controlada recebe a denominação $\mathrm{PQV}$, pois além das potências ativa e reativa, o módulo da tensão nesta barra também é especificado.

Seja um sistema genérico onde uma barra de geração $k$ do tipo P, controla o módulo de tensão na barra $l$ do tipo $\mathrm{PQV}$. Neste caso, o resíduo de potência reativa da barra $k$ torna-se uma variável dependente adicional. De modo a tornar a solução do sistema de equações possível e determinado, utiliza-se o seguinte conjunto de equações a ser utilizado a cada passo do processo iterativo:

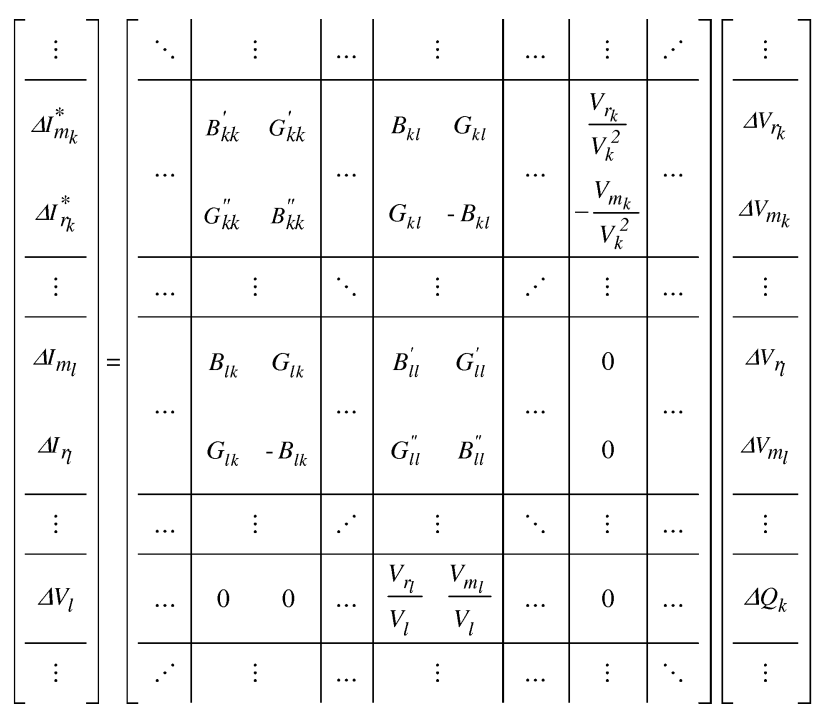

\subsection{Controle Secundário de Tensão}

Tem sido uma estratégia adotada na prevenção do colapso de tensão em regiões críticas do sistema, consistindo no ajuste coordenado da tensão em uma determinada barra, através de reguladores de tensão dos geradores, compensadores estáticos, compensadores síncronos, taps em transformadores, dentre outros.

De forma a apresentar o modelo proposto para o controle secundário de tensão, seja a Figura 1 onde $n_{g}$ geradores controlam o módulo de tensão na barra $l$ em um valor $V_{l}^{s p}$.

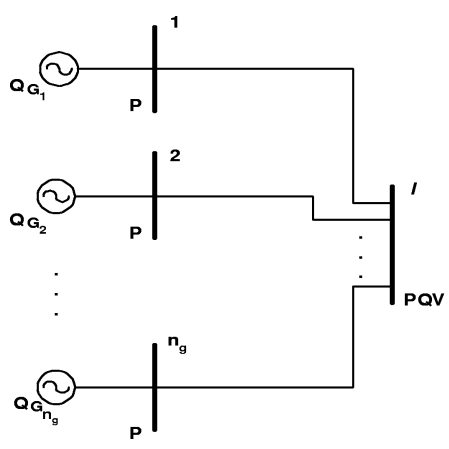

Figura 1: Múltiplos Geradores Controlando a Tensão numa Única Barra

As equações de controle a serem inseridas no problema 
de fluxo de potência são as seguintes:

$$
\begin{gathered}
Q_{G_{1}}=\alpha_{12} Q_{G_{2}} \\
Q_{G_{2}}=\alpha_{23} Q_{G_{3}} \\
\vdots \\
Q_{G_{n_{g}-1}}=\alpha_{\left(n_{g}-1\right) n_{g}} Q_{G_{n_{g}}} \\
V_{l}^{\text {calc }}-V_{l}^{s p}=0
\end{gathered}
$$

O coeficiente $\alpha_{i j}$ representa o fator de participação do gerador ou compensador síncrono da barra $i$ em relação à barra $j$, no intuito de controlar a tensão na barra controlada. Reescrevendo a Equação (14) em termos das potências líquidas injetadas nas barras, obtém-se o sistema matricial linear a ser resolvido a cada passo do processo iterativo:

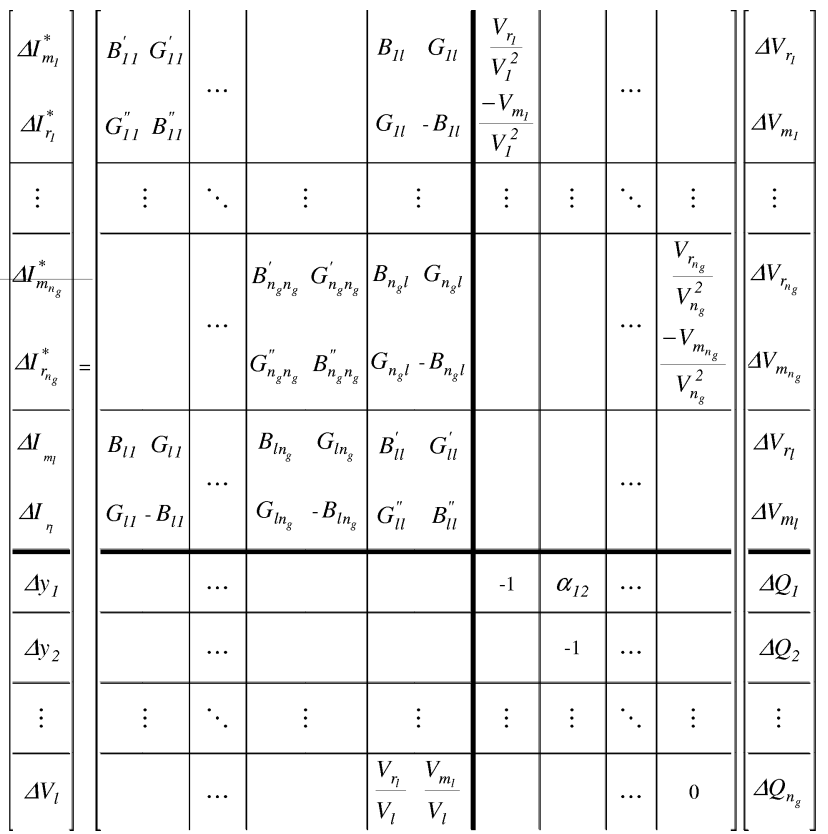

Os resíduos das equações de controle inseridas na matriz Jacobiana são dados por (4) e (17):

$$
\begin{aligned}
\Delta y_{1} & =Q_{G_{1}}-\alpha_{12} Q_{G_{2}} \\
\Delta y_{2} & =Q_{G_{2}}-\alpha_{23} Q_{G_{3}} \\
& \vdots \\
\Delta y_{\left(n_{g}-1\right)} & =Q_{G_{n_{g}-1}}-\alpha_{\left(n_{g}-1\right) n_{g}} Q_{G_{n_{g}}}
\end{aligned}
$$

Caso o controle do módulo de tensão na barra $l$ seja feito por vários transformadores, então o tap de cada um destes trans-formadores torna-se uma variável dependente adicional. As novas equações de controle inseridas no problema definem as relações entre os taps dos transformadores, bem como impõe que o valor da tensão na barra controlada seja igual ao valor especificado. De modo análogo àquele feito para os geradores, obtém-se então um sistema matricial linear a ser resolvido a cada passo do processo iterativo, possibilitando a atualização dos valores dos taps ao final de cada iteração.

\subsection{Compensador Estático de Reativos}

Este equipamento controla a tensão nodal, gerando ou absor-vendo potência reativa por meio da utilização de grupos de capacitores e indutores shunt controlados por chaveamento contínuo de tiristores. A curva característica de estado permanente de um CER está mostrada na Figura 2, onde o módulo da tensão controlada varia dentro de uma faixa. Para cada região de operação do CER há uma equação distinta de controle a ser incluída no problema de fluxo de potência.

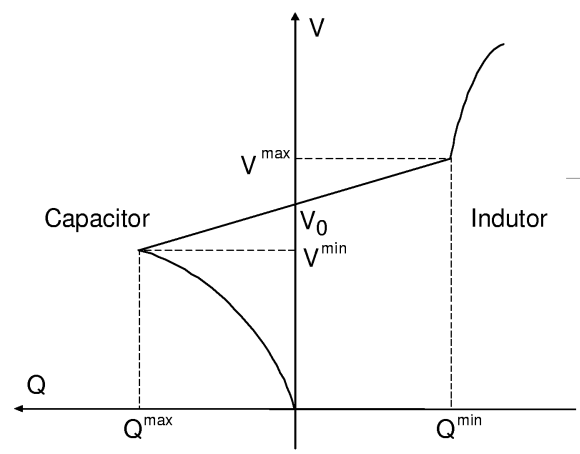

Figura 2: Tensão Versus Potência Reativa do CER

Seja um sistema genérico onde um CER localizado na barra $k$ controla o módulo de tensão na barra $l$. Do ponto de vista prático, tem-se o conhecimento da inclinação da reta de controle $r_{k}$, da tensão de referência $V_{0}$, das reatâncias mínima $B^{\min }$ e máxima $B^{\max }$. Portanto, as tensões mínima e máxima são avaliadas a cada iteração da seguinte forma:

$$
\begin{aligned}
& V_{l}^{\min }=V_{0}+r_{k} B^{\max } V_{l}^{2} \\
& V_{l}^{\max }=V_{0}+r_{k} B^{\min } V_{l}^{2}
\end{aligned}
$$

Considerando o CER na faixa linear, da Figura 2 tem-se:

$$
V_{l}=V_{0}+r_{k} Q_{G_{k}}
$$

onde $r_{k}$ e $V_{0}$ estão dados em (Da Costa et alii, 1998).

Reescrevendo a Equação (20) em função das componentes retangulares da tensão, tem-se a seguinte expressão 
linearizada a ser incorporada no problema de fluxo de potência:

$$
\Delta V_{l}^{\prime}=\frac{V_{r_{l}}}{V_{l}} \Delta V_{r_{l}}+\frac{V_{m_{l}}}{V_{l}} \Delta V_{m_{l}}+r_{k} \Delta Q_{k}
$$

onde:

$$
\Delta V_{l}^{\prime}=V_{0}+r_{k}\left(Q_{L_{k}}+Q_{k}\right)-V_{l}
$$

O sistema matricial a ser resolvido a cada passo do processo iterativo, tendo o resíduo de potência $\Delta Q_{k}$ como variável dependente adicional, é dado por:

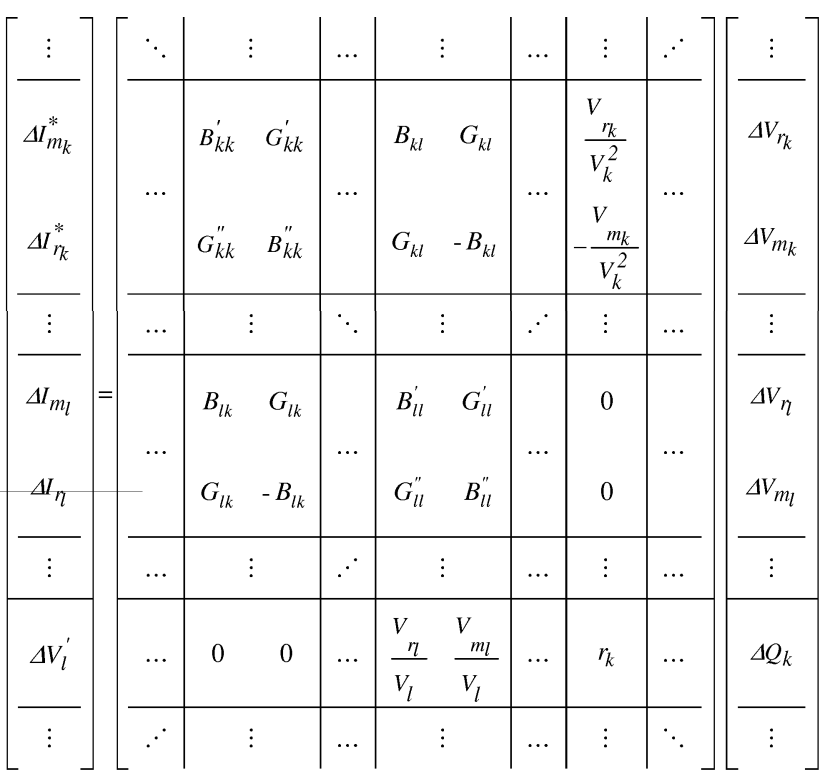

Considerando o CER operando nas faixas capacitiva e indutiva tem-se as seguintes equações, respectivamente:

$$
\begin{aligned}
Q_{G_{k}} & =\frac{Q_{G_{k}}^{\max }}{\left(V_{k}^{\min }\right)^{2}} V_{k}^{2} \\
Q_{G_{k}} & =\frac{Q_{G_{k}}^{\min }}{\left(V_{k}^{\max }\right)^{2}} V_{k}^{2}
\end{aligned}
$$

Expressando a potência gerada como função da potência líquida injetada na barra e o módulo da tensão em termos das coorde-nadas retangulares, obtém-se as equações linearizadas a serem incorporadas ao problema básico de fluxo de potência, da mesma forma como mostrado para a região linear de operação.

\section{CONTROLE DE FLUXO DE POTÊN- CIA ATIVA}

O aumento gradativo da demanda de energia nos sistemas elétricos e o conseqüente aumento da utilização dos sistemas de transmissão, impõe a necessidade de se desenvolver novas tecnologias com o intuito de controlar a potência ativa transmitida em uma linha de transmissão, tendo em vista a manutenção da estabilidade e da confiabilidade do sistema.

O ângulo de fase do transformador defasador pode ser utilizado para este tipo de controle, tornando-se uma variável depen-dente adicional. Assim, supondo um transformador defasador conectado entre as barras $k$ e $m$ de modo a controlar o fluxo de potência ativa neste ramo em um valor especificado, tem-se o conjunto de equações (28) de solução de fluxo de potência na formulação de injeção de corrente, onde:

$$
\Delta P_{k m}=P_{k m}^{s p}-P_{k m}^{c a l c}
$$

O novo ângulo de fase calculado a cada iteração é obtido por:

$$
\varphi_{k m}^{(h+1)}=\varphi_{k m}^{(h)}+\Delta \varphi_{k m}^{(h)}
$$

Uma forma alternativa de se efetuar este mesmo tipo de controle é através da utilização do compensador série controlado a tiristores (CSCT). Este dispositivo impõe

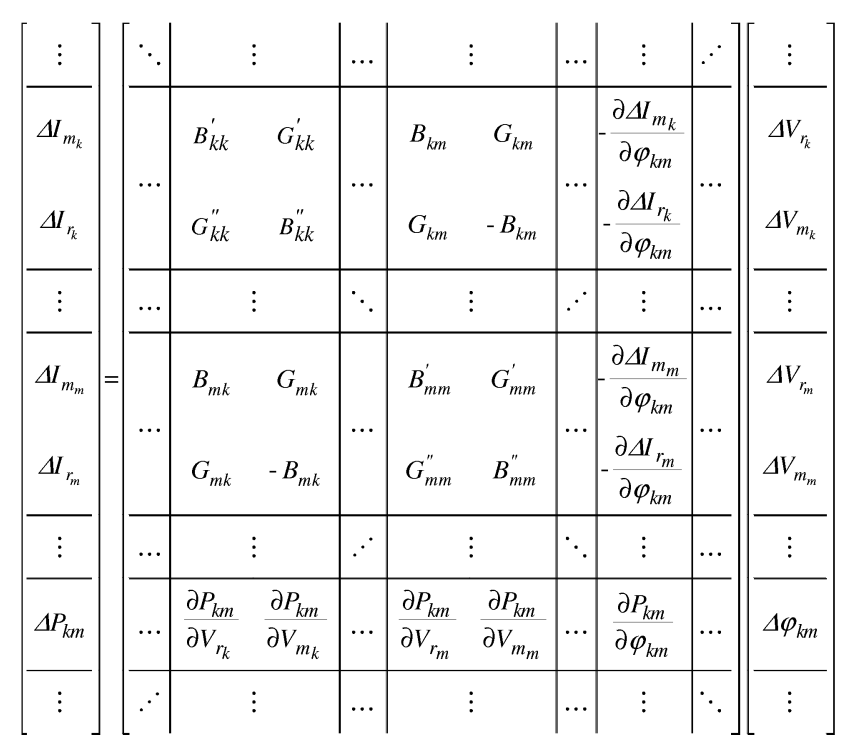

diferentes níveis de compensação série, controlando o fluxo da potência ativa numa linha através da variação de sua reatância série equivalente. Neste caso, a reatância da linha torna-se uma variável de estado adicional. Assim, o sistema matricial a ser resolvido a cada passo do processo iterativo é dado por (28) onde os termos da coluna adicional são obtidos derivando-se as equações em relação a nova variável de estado. 
A reatância série é atualizada a cada passo do processo iterativo da seguinte forma:

$$
x_{k m}^{(h+1)}=x_{k m}^{(h)}+\Delta x_{k m}^{(h)}
$$

\section{RESULTADOS}

A modelagem proposta foi implementada e testada em vários sistemas de potência sob diferentes condições. Os resultados apresentados referem-se aos sistemas testes Sudeste e Sul-Sudeste brasileiro, compostos respectivamente de 730 e 1768 barras e 1148 e 2527 ramos. Em todas as simulações realizadas, adotou-se uma tolerância de 0,01MW/MVAr. As cargas são modeladas como sendo do tipo potência constante.

\subsection{Controle de Tensão em Barras Remo- tas}

A modelagem proposta no trabalho é validada em confor-midade com os resultados apresentados na Tabela 1. Observa-se que o valor final calculado do módulo de tensão é exatamente igual ao valor especificado para o problema. Conforme mostrado na Figura 3, o resíduo máximo de potência na convergência é da ordem de $10^{-9}$ p.u.

Tabela 1: Controle de Tensão - Sistema Sul-Sudeste

\begin{tabular}{|ccccc|}
\hline $\begin{array}{c}\text { Barra de Controle (Tipo P) } \\
\text { Geração } \\
\text { (MVAr) }\end{array}$ & \multicolumn{3}{c|}{$\begin{array}{c}\text { Barra Controlada (Tipo PQV) } \\
\text { Módulo de } \\
\text { Tensão (p.u.) }\end{array}$} & $\begin{array}{c}\text { Módulo de Ten são } \\
\text { Especificado }\end{array}$ \\
\hline 28 & 49,930 & 190 & 1,0200 & 1,020 \\
42 & $-70,328$ & 211 & 1,0000 & 1,000 \\
44 & 99,460 & 178 & 1,0100 & 1,010 \\
45 & $-5,7424$ & 151 & 1,0000 & 1,000 \\
48 & $-1281,9$ & 86 & 1,0100 & 1,010 \\
55 & $-24,521$ & 215 & 1,0000 & 1,000 \\
389 & $-59,996$ & 386 & 1,0100 & 1,010 \\
400 & 128,81 & 481 & 0,9770 & 0,977 \\
401 & $-43,273$ & 480 & 0,9950 & 0,995 \\
1500 & $-8,2453$ & 1526 & 1,0200 & 1,020 \\
\hline \hline
\end{tabular}

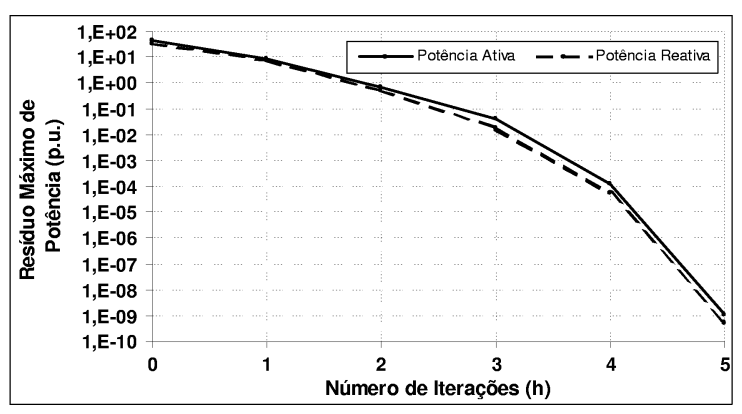

Figura 3: Característica de Convergência - Sistema SulSudeste

\subsection{Controle Secundário de Tensão}

Neste caso, os geradores das barras 403 e 404 do sistema mostrado na Figura 4, controlam o módulo da tensão na barra 483 em um valor especificado, considerando-se a relação de participação dos geradores dada por $Q_{G_{404}}=$ $1,50 Q_{G_{403}}$.

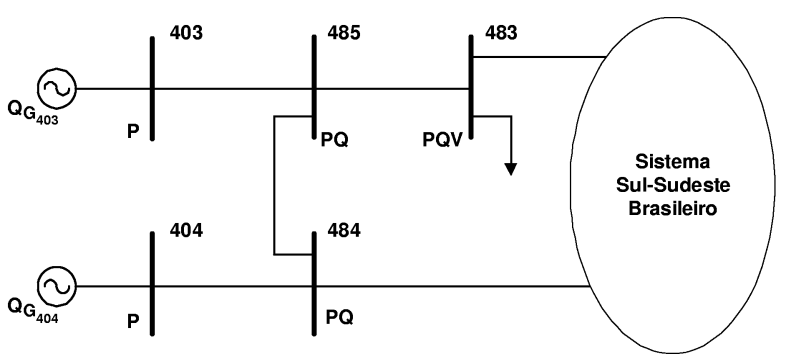

Figura 4: Controle Secundário de Tensão

A Tabela 2 mostra os resultados para sucessivos incrementos da potência da carga na barra 483. Em todas as simulações a convergência é alcançada em 5 iterações com o módulo do resíduo máximo de potência menor que $10^{-8}$ p.u.. Conforme esperado, os valores da potência reativa gerada na barra 404 são $50 \%$ maiores que os da barra 403 e os valores do módulo de tensão na barra controlada 483 são iguais ao especificado pelo controle. A Figura 5 apresenta a característica de convergência nara este disnnsitive de rentrole

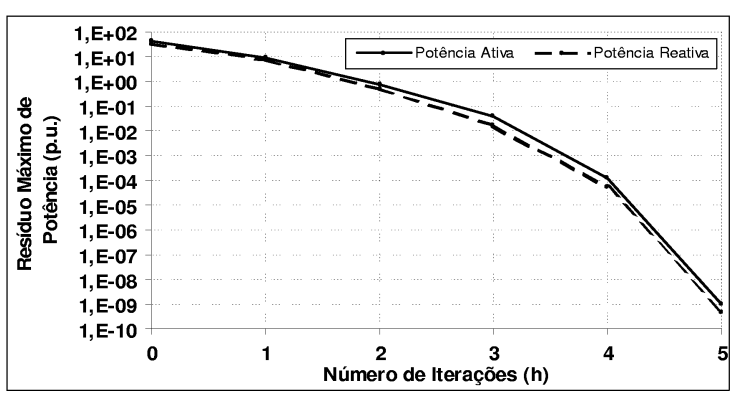

Figura 5: Convergência do Controle Secundário de Tensão

\subsection{Compensador Estático de Reativos}

Seja a representação parcial do sistema Sudeste Brasileiro ilustrado na Figura 6.3, no qual o módulo da tensão na barra 239 é controlado pelo CER conectado na barra 792, modelado pelos parâmetros $V_{0}=1,00$ p.u., $r_{k}=$ $-0,030$ p.u., $B_{\text {cap }}=0,20$ p.u. e $B_{\text {ind }}=-0,20$ p.u..

A Tabela 6.3 apresenta os resultados variando-se a po- 
Tabela 2: Resultados do Controle Secundário de Tensão

\begin{tabular}{|cccc|}
\hline \multicolumn{2}{|c|}{ Barra 483 (tipo POV) } & \multicolumn{2}{c|}{ Barras de Geracão (tipo P) } \\
Incremento de Carga & Módulo da Tensão & \multicolumn{2}{c|}{ Geração de Potência Reativa } \\
$P_{L_{483}}+j Q_{L_{483}}(\%)$ & $V_{483}$ (p.u.) & $Q_{G_{403}}$ (MVAr) & $Q_{G_{404}}$ (MVAr) \\
\hline \hline 100 & 0,97800 & 69,011 & 103,52 \\
125 & 0,97800 & 71,059 & 106,59 \\
150 & 0,97800 & 74,078 & 111,12 \\
175 & 0,97800 & 76,675 & 115,01 \\
200 & 0,97800 & 79,313 & 118,97 \\
250 & 0,97800 & 84,719 & 127,08 \\
300 & 0,97800 & 90,299 & 135,45 \\
\hline
\end{tabular}

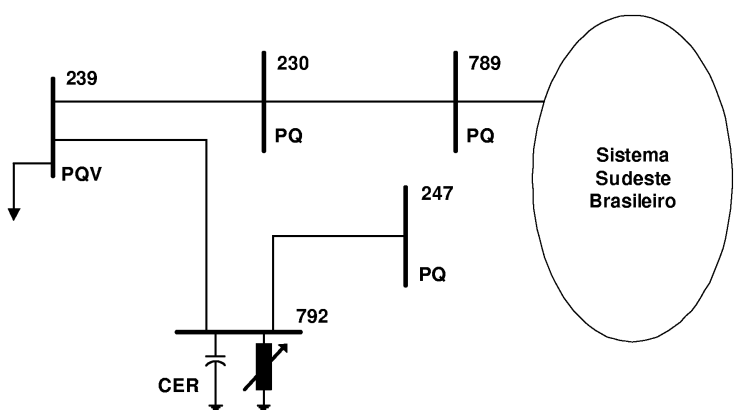

Figura 6: Subrede do Sistema Sudeste com CER

tência reativa de carga na barra com tensão controlada. Em todas as simulações a convergência do problema é alcançada em 3 ou 4 iterações com o módulo do resíduo máximo de potência menor que $10^{-5}$ p.u.

\subsection{Controle Automático de Tap em Transformadores}

Neste caso, os transformadores com ajuste automático de tap conectado entre as barras 1106 e 1107, 61 e 60, 61 e 1106 do modelo equivalente mostrado na Figura 7, controlam o módulo da tensão na barra $61 \mathrm{em}$ um valor especificado. Os parâmetros dos transformadores, bem como o fator de participação de cada um deles no controle estão dados na Tabela 4.

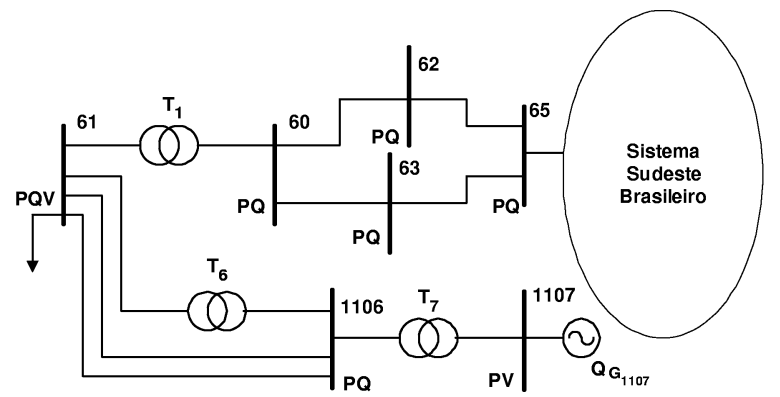

Figura 7: Representação do Sistema para Controle de Tensão
Tabela 3: Comportamento do CER Versus Potência Reativa

\begin{tabular}{|c|c|c|c|}
\hline \multicolumn{2}{|c|}{ Barra 239 (POV) } & \multicolumn{2}{|c|}{ Barra 792 (CER) } \\
\hline $\begin{array}{c}\text { Potência Reativa } \\
\text { de Carga }\end{array}$ & $\begin{array}{c}\text { Módulo } \\
\text { da Tensão }\end{array}$ & Faixa de Operação & $\begin{array}{c}\text { Geração de } \\
\text { Potência Reativa }\end{array}$ \\
\hline$Q_{L_{239}}(\mathrm{MVAr})$ & $V_{239}$ (p.u.) & & $Q_{G_{792}}$ (MVAr) \\
\hline$-60,00$ & 1,1307 & Indutiva & $-24,151$ \\
\hline$-50,00$ & 1,0914 & Indutiva & $-22,980$ \\
\hline$-40,00$ & 1,0491 & Indutiva & $-21,742$ \\
\hline$-30,00$ & 1,0059 & Linear & $-19,674$ \\
\hline$-20,00$ & 1,0021 & Linear & $-6,8357$ \\
\hline$-10,00$ & 0,99805 & Linear & 6,5018 \\
\hline 00,00 & 0,99390 & Capacitiva & 20,342 \\
\hline 10,00 & 0,93456 & Capacitiva & 20,673 \\
\hline 20,00 & 0,84688 & Capacitiva & 18,092 \\
\hline
\end{tabular}

Tabela 4: Parâmetros dos Transformadores em Estudo

\begin{tabular}{|cccccc|}
\hline $\begin{array}{c}\text { Número } \\
\text { Transformador } \\
(\#)\end{array}$ & $\begin{array}{c}\text { Barras Terminais } \\
\text { De }\end{array}$ & $\begin{array}{c}\text { Para } \\
(\mathrm{k})\end{array}$ & $\begin{array}{c}\text { Tap } \\
\text { Inicial } \\
\left(a_{\mathrm{km}}^{\text {ini }}\right)\end{array}$ & $\begin{array}{c}\text { Fator de } \\
\text { Participação } \\
(\%)\end{array}$ & $\begin{array}{c}\text { Barra } \\
\text { Controlada } \\
(\mathrm{PQV})\end{array}$ \\
\hline \hline $\mathrm{T}_{1}$ & 061 & 060 & 1,000 & 27,00 & 061 \\
$\mathrm{~T}_{6}$ & 061 & 1106 & 1,000 & 39,00 & 061 \\
$\mathrm{~T}_{7}$ & 1106 & 1107 & 1,000 & 34,00 & 061 \\
\hline \hline
\end{tabular}

A Tabela 5 apresenta os resultados da solução do problema de fluxo de potência, considerando o dispositivo de controle de tensão via tap de transformadores. A tolerância de erro de potência reativa para aplicação do controle em estudo é 1,50 p.u.. A convergência do problema é alcançada em cinco iterações.

Tabela 5: Controle de Tensão Via Múltiplos Transformadores

\begin{tabular}{|ccccc|}
\hline \multirow{2}{*}{\begin{tabular}{c} 
Número \\
\cline { 2 - 5 }$(\#)$
\end{tabular}} & $\begin{array}{c}\text { Paransformadicipaça } \\
(\%)\end{array}$ & $\begin{array}{c}\text { Tap Final } \\
\left(a_{\text {kn }}\right)\end{array}$ & $\begin{array}{c}\text { Módulo da Tensão } \\
\text { (p.u.) }\end{array}$ & $\begin{array}{c}\text { Módulo da Tensão } \\
\text { Especificada (p.u.) }\end{array}$ \\
\hline $\mathrm{T}_{1}$ & 27,00 & 0,7435 & 1,0210 & 1,021 \\
$\mathrm{~T}_{6}$ & 39,00 & 1,0739 & 1,0210 & 1,021 \\
$\mathrm{~T}_{7}$ & 34,00 & 0,9363 & 1,0210 & 1,021 \\
\hline
\end{tabular}

\subsection{Controle de Fluxo de Potência Ativa}

Foram realizadas várias simulações utilizando-se o modelo equivalente do sistema elétrico Sul-Sudeste Brasileiro. Para tanto, conecta-se no ramo entre as barras 958 e 2750 um transformador defasador com variação automática de fase, conforme ilustrado na Figura 8. Este transformador possui o tap fixo em 1,00 p.u.. Posteriormente, simulou-se este mesmo tipo de controle através de um CSCT conectado entre estas mesmas barras. Em todas as simulações o valor especificado do fluxo de potência ativa entre estas barras é 26,91 MW.

As Tabelas 6 e 7 mostram os resultados da solução do fluxo de potência do sistema ilustrado na Figura 8, para 


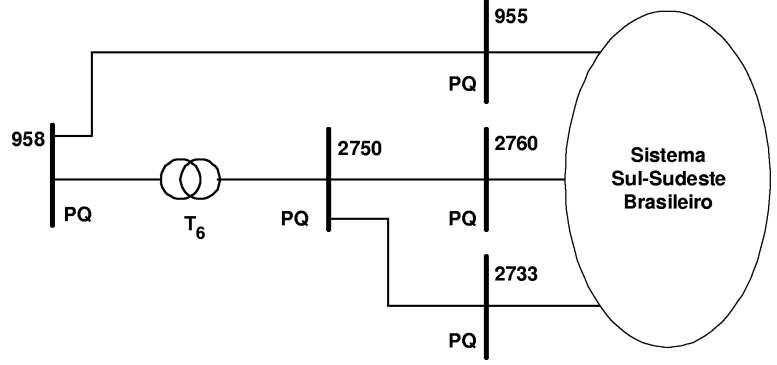

Figura 8: Sistema para Controle de Fluxo de Potência

diversos níveis de carregamento da potência de carga na barra 2750. Nas simulações com o transformador defasador, o seu valor inicial do ângulo de fase do é $0,100^{\circ}$. Nas simulações com CSCT, o valor inicial da reatância equivalente da linha é igual a $6,12 \%$. A convergência do problema em todos os casos é alcançada em 5 iterações com o módulo do resíduo máximo de potência menor que $10^{-7}$ p.u..

Tabela 6: Ângulos de Fase do Transformador

\begin{tabular}{|c|c|c|c|c|c|}
\hline \multicolumn{2}{|c|}{ Potência da Carga } & \multirow{2}{*}{\multicolumn{2}{|c|}{$\frac{\frac{\text { Módulo }}{\text { da Tensão }}}{\text { (p.u.) }}$}} & \multirow{3}{*}{$\frac{\hat{\text { Angulo de Fase do }}}{\frac{\text { Transformador }}{\text { (graus) }}}$} & \multirow{3}{*}{$\begin{array}{c}\frac{\text { Fluxo de }}{\text { Potência Ativa }} \\
\text { (MW) } \\
P_{958-2750}\end{array}$} \\
\hline \multirow{2}{*}{$\begin{array}{c}\text { Ativa } \\
\text { (MW) } \\
\left(P_{L_{2750}}\right) \\
\end{array}$} & \multirow{2}{*}{$\begin{array}{c}\text { Reativa } \\
\text { (MVAr) } \\
\left(Q_{L_{2750}}\right)\end{array}$} & & & & \\
\hline & & $\left(V_{958}\right)$ & $\left(V_{2750}\right)$ & & \\
\hline 0,0 & 0,0 & 0,99822 & 1,0 & $-0,000$ & 26,910 \\
\hline 20,0 & 20,0 & 0,98286 & 0,97836 & & 26 \\
\hline 40,0 & 40 & 0,96632 & & & \\
\hline 60,0 & 60 & 4835 & & & 26, \\
\hline 80,0 & 80,0 & 0,92858 & 0,89555 & $-6,249$ & 26,910 \\
\hline 100,0 & 100,0 & 0,90645 & 0,86197 & $-8,230$ & 26,910 \\
\hline
\end{tabular}

Tabela 7: Compensação Série Controlada a Tiristores

\begin{tabular}{|c|c|c|c|c|c|c|c|}
\hline \multicolumn{2}{|c|}{ Barra } & \multicolumn{2}{|c|}{$\begin{array}{l}\text { Potência } \\
\text { da carga }\end{array}$} & \multicolumn{2}{|c|}{$\frac{\text { Reatância Equivalente }}{\underline{\text { da Linha }(\%)}}$} & \multicolumn{2}{|c|}{$\frac{\text { Fluxo de Potência }}{\underline{\text { Ativa (MW) }}}$} \\
\hline (k) & (m) & $P_{L_{m}}$ & $Q_{L_{m}}$ & Inicial & Final & $\left(P_{k m}\right)$ & $\left(P_{k m}^{s p}\right)$ \\
\hline 958 & 2750 & $\overline{0,0}$ & $\overline{0,0}$ & $\overline{6,12}$ & $\overline{6,12}$ & 26,910 & 26,91 \\
\hline 958 & 2750 & 20,0 & 20,0 & 6,12 & 14,625 & 26,910 & 26,91 \\
\hline 958 & 2750 & 40,0 & 40,0 & 6,12 & 21,972 & 26,910 & 26,91 \\
\hline 958 & 2750 & 60,0 & 60,0 & 6,12 & 28,726 & 26,910 & 26,91 \\
\hline 958 & 2750 & 80,0 & 80,0 & 6,12 & 35,068 & 26,910 & 26,91 \\
\hline 958 & 2750 & 100,0 & 100,0 & 6,12 & 41,044 & 26,910 & 26,91 \\
\hline
\end{tabular}

Observa-se que o ângulo de fase do defasador e a reatância equivalente ajustam-se à medida que o carregamento da barra 2750 é variado, de modo a controlar o fluxo de potência entre as barras terminais no valor especificado. Portanto, os resultados apresentados validam a formulação proposta.

\section{CONCLUSÕES}

Este trabalho apresenta o desenvolvimento e a implementação de modelagens matemáticas referentes aos di- versos dispositivos de controle no problema de fluxo de potência. Para isto, utiliza o método iterativo de Newton Raphson aplicado às equações de injeção de corrente, obtendo-se uma matriz Jaco-biana altamente esparsa e facilmente calculada a cada iteração, uma vez que todos os elementos fora dos blocos diagonais são exatamente iguais aos termos correspondentes da matriz admitância nodal.

Outro mérito a se destacar está no fato de que a inclusão das equações referentes aos dispositivos de controle, não altera os elementos e a estrutura da matriz Jacobiana original. Desta forma, tem-se grande facilidade e flexibilidade na incorporação e retirada das equações de controle durante o processo iterativo, bem como permite a inclusão de modelagens mais realísticas dos componentes utilizados nos sistemas de potência.

Os resultados apresentados demonstram a validade dos modelos matemáticos desenvolvidos. Assim sendo, as formulações e os modelos dos controles propostos constituem-se em valiosas ferramentas na solução e análise dos problemas atuais de fluxo de potência, decorrentes do crescente aumento da topologia e demanda de energia elétrica das redes e da conseqüente operação dos sistemas elétricos de potência muito próxima de seus limites.

\section{REFERÊNCIAS}

Bacher R. and W.F. Tinney. (Outubro, 1989). Faster local power flow solutions: The zero mismatch approach. IEEE Transactions on Power Systems, 4, No. 4:1345-1354.

Da Costa V.M., J.L.R. Pereira, and N. Martins. (Abril, 1998). Modelagem de dispositivos de controle numa formulação aumentada para fluxo de potência. Revista da Sociedade Brasileira de Automática, 9, No. 1.

Da Costa V.M., N. Martins, and J.L.R. Pereira. (Novembro, 1999). Developments in the Newton Raphson power flow formulation based on current injections. IEEE Transactions on Power Systems, 14, No. 4:1320-1326.

Fuerte-Esquivel C.R., and E. Acha. (Novembro,1997). A Newton type algorithm for the control of power flow in electrical power networks. IEEE Transactions on Power Systems, 12, No. 4:1474-1480.

Garcia, P.A.N., J.L.R. Pereira, S. Carneiro Jr, V.M. Da Costa and N. Martins. (Maio, 2000). Three-phase power flow calculations using the current injection 
method. IEEE Transactions on Power Systems, 15, No. 2:508-514.

Gotham D.J., and G.T. Heydt. (Fevereiro, 1998). Power flow control and power flow studies for systems with FACTS devices. IEEE Transactions on Power Systems,13, No. 1:60-65.

Monticelli A.J., A Garcia, and O.R. Saavedra. (Novembro, 1990). Fast decoupled load flow: Hypothesis, derivations, and testing. IEEE Transactions on Power Systems, 5, No. 4:1425-1431.

Semlyen A. (Agosto, 1996). Fundamental concepts of a Krylov subspace power flow methodology. IEEE Transactions on Power Systems,11, No. 3:15281537.

Talaq J. (Julho, 1994). Modelling and elimination of load buses in power flow solutions. IEEE Summer Power Meeting, Paper 94 SM 505-8 PWRS San Francisco. 